\title{
Bilateral Structural Network Abnormalities in Epilepsy Associated With Bottom-of-Sulcus Dysplasia
}

\author{
Remika Mito, PhD, David N. Vaughan, MBBS, PhD, Mira Semmelroch, MSc, Alan Connelly, PhD, and \\ Graeme D. Jackson, MD, FRACP
}

Neurology ${ }^{\circledR}$ 2022;98:e152-e163. doi:10.1212/WNL.0000000000013006

\section{Abstract}

\section{Background and Objectives}

To identify white matter fiber tracts that exhibit structural abnormality in patients with bottomof-sulcus dysplasia (BOSD) and investigate their association with seizure activity.

\section{Methods}

Whole-brain fixel-based analysis of diffusion MRI data was performed to identify white matter fiber tracts with significant reductions in fiber density and cross-section in patients with BOSD $(n=20)$ when compared to healthy control participants $(n=40)$. Results from whole-brain analysis were used to investigate the association of fiber tract abnormality with seizure frequency and epilepsy duration.

\section{Results}

Despite the focal nature of the dysplasia, patients with BOSD showed widespread abnormality in white matter fiber tracts, including the bilateral corticospinal, corticothalamic, and cerebellothalamic tracts, superior longitudinal fasciculi, corpus callosum (body), and the forceps major. This pattern of bilateral connectivity reduction was not related to the laterality of the lesion. Exploratory post hoc analyses showed that high seizure frequency was associated with greater reduction in fiber density at the forceps major, bilateral corticospinal, and cerebellothalamic tracts.

\section{Discussion}

We demonstrate evidence of a bilaterally distributed, specific white matter network that is vulnerable to disruption in BOSD. The degree of tract abnormality is partly related to seizure activity, but additional contributors such as the genetic background and effects of treatment or environment have not been excluded.

\author{
Correspondence \\ Dr. Mito \\ remika.mito@florey.edu.au
}

MORE ONLINE

A CME Course

NPub.org/cmelist

From the Florey Institute of Neuroscience and Mental Health (R.M., D.N.V., M.S., A.C., G.D.J.), Heidelberg; Florey Department of Neuroscience and Mental Health (D.N.V., A.C., G.D.J.), University of Melbourne; and Department of Neurology (D.N.V., G.D.J.), Austin Health, Heidelberg, Australia.

Go to Neurology.org/N for full disclosures. Funding information and disclosures deemed relevant by the authors, if any, are provided at the end of the article. 


\section{Glossary}

BOSD = bottom-of-sulcus dysplasia; $\mathbf{C F E}=$ connectivity-based fixel enhancement; DWI = diffusion-weighted imaging; FBA = fixel-based analysis; FC = fiber bundle cross-section; FCD = focal cortical dysplasia; FD = fiber density; FDC = fiber density and cross-section; FDR = false discovery rate; FOD = fiber orientation distribution; FWE = family-wise error; TE = echo time; TR = repetition time.

Focal epilepsies are widely viewed as network disorders whereby focal lesions can drive extensive abnormalities within brain networks. ${ }^{1,2}$ These network conceptualizations of epilepsy are predominantly driven by evidence from temporal lobe epilepsy; however, recent work suggests that focal cortical dysplasia (FCD) may also be characterized by widespread functional network abnormalities. ${ }^{3,4}$ It remains unclear whether there are specific structural networks that are vulnerable to disruption in FCD, and efforts to probe networkwide disruptions have to some extent been hampered by the heterogeneity of these epileptogenic lesions.

Bottom-of-sulcus dysplasia (BOSD) is a type of FCD with distinctive neuropathologic and neuroimaging hallmarks. ${ }^{5-7}$ Given the excellent postsurgical outcomes after their complete resection, ${ }^{6,7}$ BOSD lesions are accepted to be intrinsically epileptogenic and are considered focal abnormalities, rather than pervasive lesions. However, like other forms of focal epilepsy, BOSD could be associated with more widespread disruption to brain organization. It remains unclear whether BOSD is characterized by disruption to specific brain networks, and if so, what structural networks are affected.

In this study, we explored whether patients with intractable epilepsy due to BOSD exhibit disruptions to structural brain networks when compared to healthy control participants. To this end, we applied a technique known as fixel-based analysis (FBA), ${ }^{8}$ which enables identification of specific white matter structures that exhibit clinically important reductions in structural connectivity. We investigated tract-specific group differences across the whole brain and performed exploratory post hoc analyses to probe the relationship of tract disruption with seizure frequency and disease duration.

\section{Methods}

\section{Participants}

Patients with focal epilepsy due to cortical dysplasia were identified from the Austin Hospital Comprehensive Epilepsy Program, Melbourne, Australia, between 2008 and 2019. Only participants who had undergone a preoperative MRI scan at the Florey Institute of Neuroscience and Mental Health, which included high angular resolution diffusion-weighted imaging (DWI) acquisition, were included. Preoperative MRI scans were reviewed for all ascertained patients by expert neurologists (D.N.V., G.D.J.).

A diagnosis of BOSD was based on MRI scans showing a focal lesion at the bottom of a sulcus, with cortical thickening and gray-white matter blurring being maximal at the depth of the sulcus. Only patients with a single BOSD confined to 1 sulcus were included. For those patients who had undergone surgery and had histopathology performed, only patients with FCD type II histopathology were included. Patients with intellectual disability were excluded from this cohort.

Twenty patients met inclusion criteria ( 12 male, 8 female), with a mean age of 32.7 years (range 15-53 years). Seventeen patients $(17 / 20$ [85\%]) had subsequently undergone surgical resection, and FCD type II was confirmed by histopathology in all. Complete seizure freedom was achieved in 14/17 $(82 \%)$ of those who underwent surgery, with minor attacks reported in the remaining patients likely attributable to incomplete resection. BOSD lesions were in the right hemisphere in $13 / 20(65 \%)$ and left hemisphere in $7 / 20(35 \%)$. BOSDs were located in the frontal lobe in 12/20 (60\%) patients, parietal lobe in 6/20 (30\%), occipital lobe in $1 / 20$ (5\%), and temporal lobe in $1 / 20(5 \%)$.

Healthy control participants were selected from previously acquired DWI control data obtained at our institute. For each patient, 2 healthy control participants were selected, who matched the patient in age, sex, and scanner on which MRI was acquired, resulting in 40 control participants ( 24 male, 16 female), with a mean age of 33.1 years (range 14-55).

\section{Standard Protocol Approvals, Registrations, and Patient Consents}

All participants provided informed written consent and the study was approved by the ethics committee at Austin Health (HREC number H2013/05123).

\section{MRI Data Acquisition}

MRI data were acquired at 3T on either a Siemens Tim Trio with a 12-channel head coil receiver or Siemens Skyra with a 32-channel head coil receiver. Echoplanar imaging DWI data were acquired on the Skyra with 1 of the following parameter sets: (1) 60 axial slices, repetition time (TR)/echo time (TE) $8,400 / 110 \mathrm{~ms}, 2.5 \mathrm{~mm}$ isotropic voxels, 64 diffusion-weighted images $\left(b=3,000\right.$ seconds $\left./ \mathrm{mm}^{2}\right)$, and $8 \mathrm{~b}=0$ images; (2) 60 axial slices, TR/TE $8,800 / 110 \mathrm{~ms}, 2.5 \mathrm{~mm}$ isotropic voxels, 64 diffusion-weighted images $\left(b=3,000\right.$ seconds $\left./ \mathrm{mm}^{2}\right)$, and 1 $\mathrm{b}=0$ image. Equivalent DWI data were acquired on the Trio with the following parameters: 60 axial slices, TR/TE 8,300/ $110 \mathrm{~ms}, 2.5 \mathrm{~mm}$ isotropic voxels, 60 diffusion-weighted images $\left(b=3,000\right.$ seconds $\left./ \mathrm{mm}^{2}\right)$, and $8 b=0$ images. Sixteen patients with BOSD and 32 matched control participants were scanned on the Skyra; 4 patients and the 8 matched 
Table 1 Clinical Characteristics of Patients With Bottom-of-Sulcus Dysplasia

\begin{tabular}{|c|c|c|c|c|c|c|c|c|}
\hline Case & Sex & $\begin{array}{l}\text { Age at } \\
\text { scan, y }\end{array}$ & Pathology & Lesion location & $\begin{array}{l}\text { Age at seizure } \\
\text { onset, } y\end{array}$ & $\begin{array}{l}\text { Disease } \\
\text { duration, y }\end{array}$ & $\begin{array}{l}\text { Seizure frequency } \\
\text { category }^{\text {a }}\end{array}$ & Scanner \\
\hline 1 & M & 18 & FCD type $2 A$ & R precentral sulcus & 1 & 17 & Medium & Skyra \\
\hline 2 & M & 52 & FCD type 2B & $\mathrm{R}$ inferior frontal gyrus & 18 & 34 & High & Trio \\
\hline 3 & $\mathrm{~F}$ & 34 & FCD type $2 A$ & $\mathrm{R}$ parietal precuneus & 3 & 31 & Medium & Skyra \\
\hline 4 & M & 25 & FCD type 2B & R postcentral gyrus & 18 & 7 & High & Skyra \\
\hline 5 & M & 53 & FCD type $2 B$ & L cingulate sulcus posterior frontal & 4 & 49 & High & Skyra \\
\hline 6 & M & 49 & FCD type $2 B$ & $\mathrm{~L}$ anterior inferior frontal/frontal pole & 15 & 34 & Low & Skyra \\
\hline 7 & M & 15 & FCD type $2 B$ & $L$ inferior frontal & 5 & 10 & Low & Skyra \\
\hline 8 & M & 25 & FCD type $2 B$ & R precuneus (medial sulcus) & 5 & 20 & Low & Skyra \\
\hline 9 & M & 29 & NA & L supramarginal gyrus & 24 & 5 & Low & Skyra \\
\hline 10 & $\mathrm{~F}$ & 24 & FCD type $2 B$ & $\mathrm{R}$ frontal cingulate gyrus & 7 & 17 & High & Skyra \\
\hline 11 & $\mathrm{~F}$ & 45 & NA & R superior frontal sulcus & 19 & 26 & Medium & Skyra \\
\hline 12 & $\mathrm{~F}$ & 17 & $\begin{array}{l}\text { FCD type } 2 \mathrm{~A} \text { (limited } \\
\text { sample) }\end{array}$ & $\mathrm{R}$ postcentral sulcus & 12 & 5 & Low & Skyra \\
\hline 13 & M & 38 & FCD type $2 B$ & L middle frontal gyrus, superior frontal & 9 & 29 & High & Trio \\
\hline 14 & $\mathrm{~F}$ & 23 & FCD type $2 B$ & R superior frontal gyrus & 13 & 10 & High & Skyra \\
\hline 15 & M & 32 & FCD type $2 B$ & $\begin{array}{l}\mathrm{R} \text { anterior frontal (middle and inferior } \\
\text { frontal junction) }\end{array}$ & 3 & 29 & High & Skyra \\
\hline 16 & M & 29 & FCD type $2 B$ & L occipital (lingual and fusiform gyri) & 5 & 24 & Low & Skyra \\
\hline 17 & $\mathrm{~F}$ & 43 & NA & $\mathrm{R}$ anterior superior frontal & 3 & 39 & Medium & Skyra \\
\hline 18 & $\mathrm{~F}$ & 37 & FCD type $2 B$ & $\begin{array}{l}\text { R parieto-occipital, back margin of } \\
\text { sylvian }\end{array}$ & 18 & 19 & Medium & Trio \\
\hline 19 & $\mathrm{~F}$ & 38 & FCD type $2 A$ & R superior frontal gyrus & 4 & 34 & Low & Skyra \\
\hline 20 & M & 21 & N/A & R fusiform & 10 & 11 & Medium & Trio \\
\hline
\end{tabular}

Abbreviation: $F C D=$ focal cortical dysplasia.

${ }^{a}$ Seizure frequency was patient-reported and computed into seizures per month. For post hoc analyses, seizure frequency was categorized into low ( $<10$ seizures per month), medium (10-80 seizures per month), and high ( $>80$ seizures per month) categories.

controls were scanned on the Trio. Scanner information for each patient is available in Table 1 .

Isotropic T1-weighted magnetization-prepared rapid acquisition gradient echo (MPRAGE) images were also acquired from all participants and used to compute intracranial volume using SPM8.

\section{DWI Processing}

All DWI data were preprocessed and analyzed using MRtrix $3{ }^{9}$

Diffusion-weighted images were preprocessed with the following steps: denoising, Gibbs ringing removal, eddy current correction and motion correction, bias field correction, and upsampling to a voxel size of $1.3 \mathrm{~mm}^{2}$.

Fiber orientation distribution (FOD) functions were subsequently computed using single-shell, 3-tissue constrained spherical deconvolution $\left(\right.$ SS3T-CSD $\left.{ }^{10}\right)$, with group-averaged response functions for white matter, gray matter, and CSF, using MRtrix3Tissue. Joint bias field and intensity normalization were then performed. Patients with left-sided BOSD had their FOD images flipped left to right, to align epileptic side across all patients. For each patient who was flipped, the 2 matched control participants also had their FOD images flipped left to right. We also performed analyses using unflipped FOD images to determine whether there were any differences between patients with BOSD and controls irrespective of lesion hemisphere.

A symmetrical study-specific FOD template was generated from a subset of participants (10 BOSD, 10 controls), who were randomly selected from the study cohort. FOD images from these 20 participants, along with their left-to-right flipped images, were used to generate a population template image using an iterative registration and averaging approach. ${ }^{11}$ FOD 
Figure 1 Fiber Tracts Exhibiting Significant Reductions in Patients With Bottom-of-Sulcus Dysplasia (BOSD) With Aligned Lesion Hemisphere

A. Fiber density (FD)

\section{Patients with BOSD < controls}

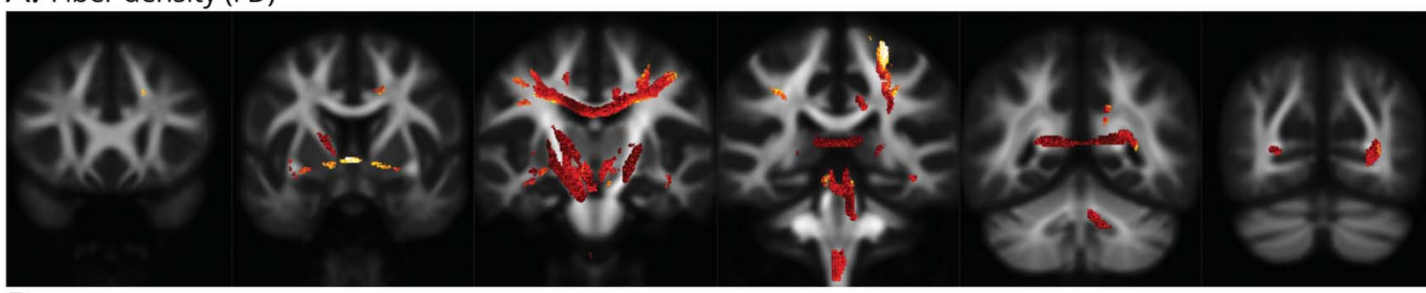

B. Fiber cross section (FC)

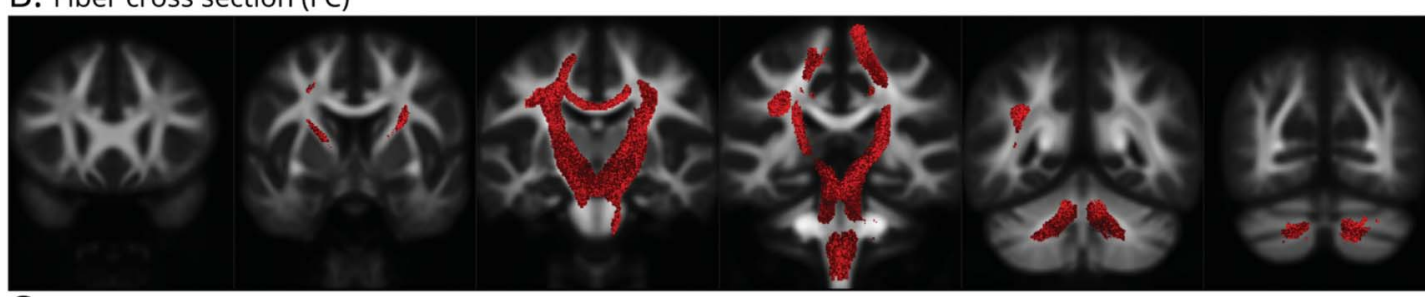

C. Fiber density and cross section (FDC)

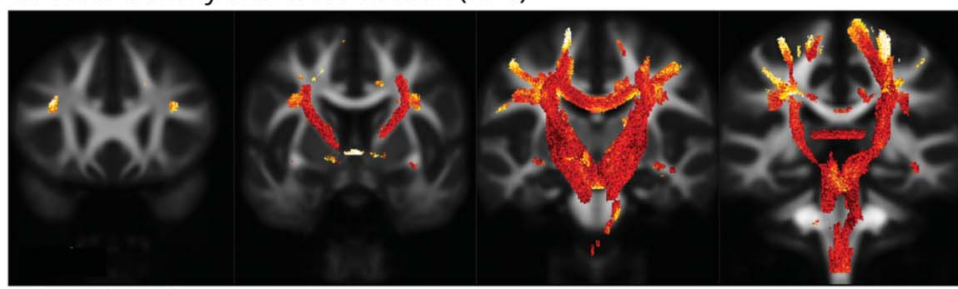

Contralateral

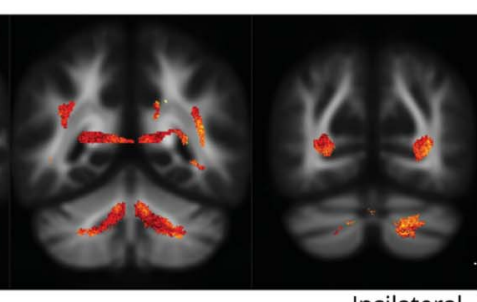

Ipsilateral

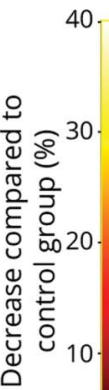

0

Fiber pathways exhibiting significant reductions in fiber density (FD; A), fiber bundle cross-section (FC; B), and fiber density and cross-section (FDC; C) in patients with BOSD compared to control participants are displayed on coronal slices at 18-mm increments. Significant results from whole-brain fixel-based analysis are shown on streamline segments that have been cropped from the template tractogram to include only streamline points that correspond to significant fixels (family-wise error-corrected $p$ value $<0.05$ ). Streamlines are colored by percentage decrease in patients with BOSD compared to controls.

images from all participants were then registered to this unbiased symmetrical template using FOD-guided nonlinear registration. ${ }^{11}$

Whole-brain fiber tracking (a tractogram) was generated using probabilistic tractography on the population template image to generate 20 million streamlines that were subsequently filtered to 2 million streamlines using the SIFT algorithm. ${ }^{12}$

We applied the FBA ${ }^{8}$ framework, where the term "fixel" refers to a specific population of fibers within a single image voxel. In this context, quantitative measures are not voxel-averaged, but are rather derived at each fixel, resulting in measures that are specific to the fiber pathways (or tracts) directed at a particular orientation, even when multiple crossing fiber tracts pass through a given voxel. Measures of apparent fiber density (FD), fiber bundle cross-section (FC), and a combined metric of fiber density and cross-section (FDC) were obtained for each participant at each white matter fixel. In brief, FD was computed according to the Apparent Fiber Density framework, ${ }^{13}$ by which a quantitative measure of fiber density can be derived from FOD images, given that the integral over each lobe of the FOD is proportional to the intra-axonal volume of axons aligned in that direction. The FD value for each fixel in each participant was assigned to a fixel template mask following spatial normalization. Morphologic changes in the cross-section of a fiber pathway were encapsulated by the FC metric, ${ }^{8}$ by using the nonlinear warps to compute the change in fiber bundle cross-section required to spatially normalize the participant image to the template image. The FD and FC metrics were multiplied to obtain the FDC metric, which combines both sources of information to characterize total change to specific white matter pathways. The FDC metric is considered the most robust measure with which to probe fiber tract changes; however, biologically useful information can be provided by also investigating the FD and FC metrics separately. ${ }^{8}$ For this reason, we investigated all 3 fixel-based metrics in this study, although it should be noted that FD and FC are not entirely independent measures. ${ }^{8}$

Fixels that exhibited a significant decrease in FDC in the BOSD group when compared to controls were extracted for the purposes of exploratory post hoc analyses. To delineate the white matter tracts to which these fixels belonged, we first assigned significant fixels from our whole-brain analysis to a population template tractogram, then consulted the JHU white matter tractography atlases ${ }^{14}$ to determine fiber pathways to which the 
Figure 2 Fiber Tracts Exhibiting Significant Fiber Density and Cross-section (FDC) Reductions in Bottom-of-Sulcus Dysplasia (BOSD) and Their Association With Seizure Frequency and Disease Duration
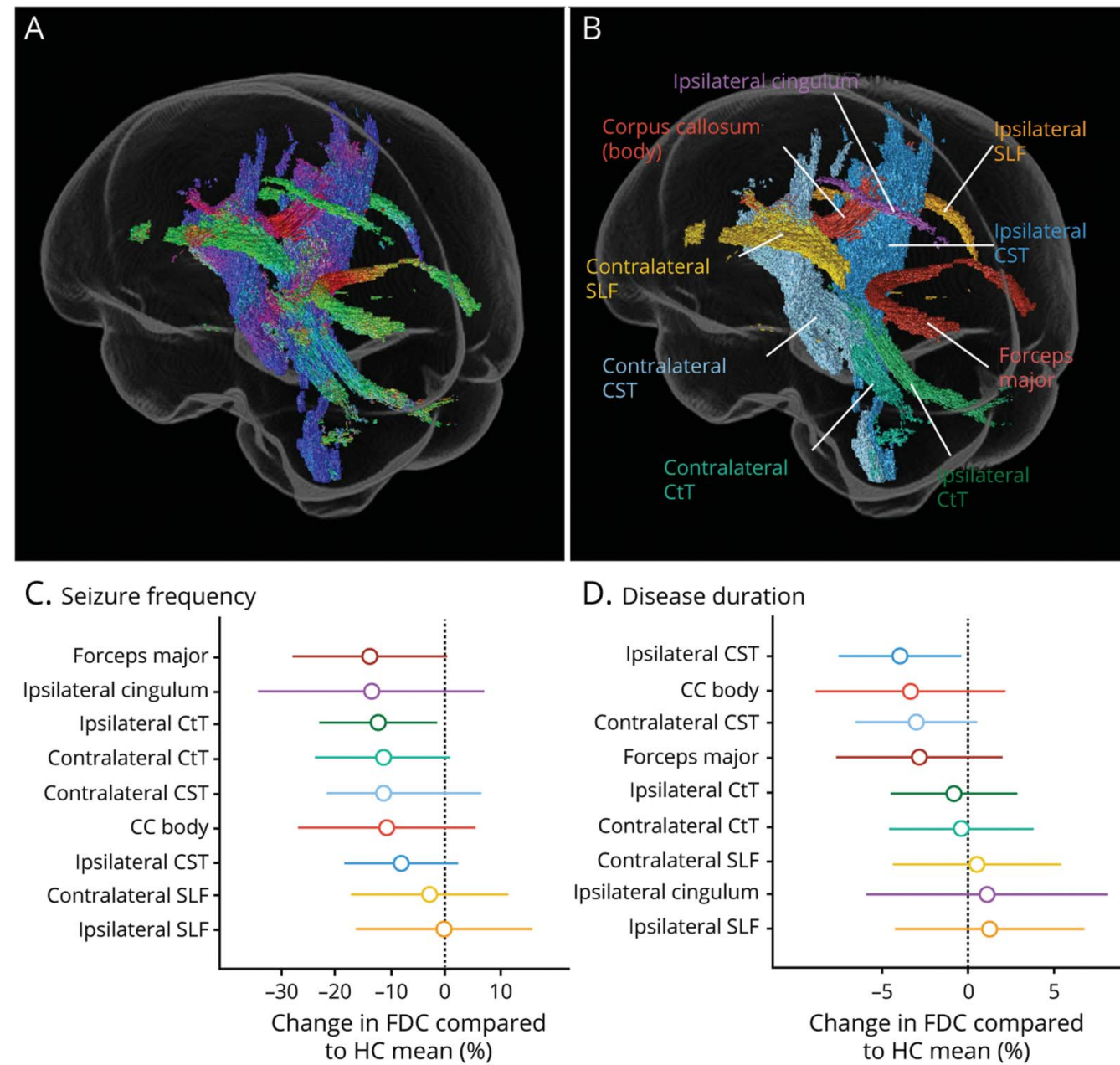

D. Disease duration

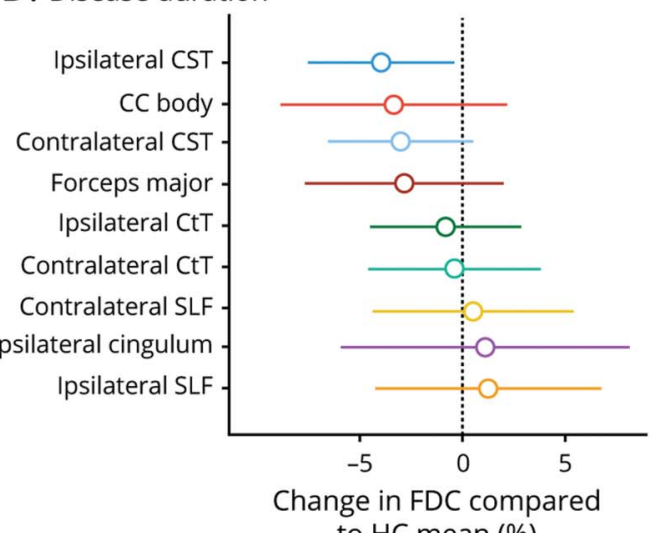

The streamlines corresponding to fixels that exhibited significant reductions in FDC in the BOSD group (Figure 1C) are shown in a glass brain representation to demonstrate the 3D extent of structural white matter abnormalities. These streamlines are colored by direction in (A) (blue: superior-inferior; green: anteriorposterior; red: left-right). The same fiber structures are shown in (B), this time colored by the fiber tract to which they belong. Nine fiber tracts were identified and post hoc comparisons with clinical metrics were performed by extracting mean fixel-based metrics within each of this fiber tracts. Effect sizes from these linear models are shown for each tract in the bottom 2 panels, with bars representing 95\% confidence intervals. (C) The effect sizes reflect the percentage change in FDC (compared to the healthy control $[\mathrm{HC}]$ mean) in each given tract going between low and high seizure frequency categories. The tracts are ordered by effect size (largest to smallest effect). The largest effect of seizure frequency on tract FDC was observed in the forceps major. (D) Effect sizes are shown for each tract and reflect the percentage change in FDC (compared to the $\mathrm{HC}$ mean) for that tract per 10 years of disease duration. The largest effect of disease duration on tract FDC was observed in the corticospinal tracts (CSTs). CC = corpus callosum; CtT = cerebellothalamic tract; SLF = superior longitudinal fasciculus.

identified tract trajectories could be assigned, using manually drawn inclusion and exclusion regions of interest.

\section{Statistical Analysis}

Whole-brain fixel-based analyses of fixel-based metrics (FD, FC, and FDC) were performed using a general linear model, comparing patients with BOSD to controls, with age, intracranial volume, and scanner type included as nuisance covariates. In this study, we use the term "whole-brain fixelbased analysis" to refer to a comparison of all white matter fixels identified within the population template brain. Connectivitybased smoothing and statistical inference were performed with connectivity-based fixel enhancement (CFE), using the streamlines from the template tractogram and default CFE parameters. ${ }^{15}$ Family-wise error (FWE) corrected $p$ values were then assigned to each fixel using nonparametric permutation testing over 5,000 permutations.

In addition to the primary whole-brain fixel-based analyses in this study, in which we aligned lesion hemisphere across all patients, we also performed whole-brain FBA comparing patients with BOSD to controls without aligning lesion side. We additionally compared patients with BOSD with frontal vs parietal lesions to each other and to controls, to explore potential lobar-specific findings in our cohort. The methodology and results for these analyses are described in the Supplement.

We subsequently categorized fixels that exhibited significant FDC decrease in the BOSD group compared to controls from our primary whole-brain analyses (where lesion hemisphere 
Table 2 Effect of Seizure Frequency and Disease Duration on Tract-Specific Fiber Density and Cross-section (FDC)

\begin{tabular}{|c|c|c|c|c|}
\hline \multirow[b]{2}{*}{ Tract } & \multicolumn{2}{|l|}{ Seizure frequency } & \multicolumn{2}{|l|}{ Disease duration } \\
\hline & $\%$ Change in FDC compared to $\mathrm{HC}$, mean $(95 \% \mathrm{CI})$ & $p$ Value & \% Change in FDC compared to $\mathrm{HC}$, mean $(95 \% \mathrm{CI})$ & $p$ Value \\
\hline Forceps major & $-13.85(-27.97$ to 0.27$)$ & 0.054 & $-2.8(-7.67$ to 2.04$)$ & 0.236 \\
\hline CC body & $-10.70(-26.80$ to 5.41$)$ & 0.177 & $-3.3(-8.87$ to 2.21$)$ & 0.219 \\
\hline Ipsilateral CST & $-8.13(-18.50$ to 2.24$)$ & 0.115 & $-4.0(-7.52$ to -0.38$)$ & 0.032 \\
\hline Contralateral CST & $-11.33(-21.66$ to -1.00$)$ & 0.033 & $-3.0(-6.55$ to 0.56$)$ & 0.092 \\
\hline Ipsilateral CtT & $-12.25(-22.98$ to -1.53$)$ & 0.028 & $-0.79(-4.48$ to 2.90$)$ & 0.654 \\
\hline Contralateral CtT & $-11.89(-25.22$ to 1.43$)$ & 0.0765 & $-0.27(-4.85$ to 14.3$)$ & 0.902 \\
\hline Ipsilateral SLF & $0.399(-16.48$ to 15.68$)$ & 0.958 & $1.29(-4.25$ to 6.82$)$ & 0.628 \\
\hline Contralateral SLF & $-3.024(-17.36$ to 11.31$)$ & 0.659 & $0.53(-4.40$ to 5.46$)$ & 0.822 \\
\hline Ipsilateral cingulum & $-13.53(-34.09$ to 7.03$)$ & 0.181 & $1.13(-5.95$ to 8.20$)$ & 0.739 \\
\hline
\end{tabular}

Abbreviations: CC = corpus callosum; CST = corticospinal tract; CtT = cerebellothalamic tract; SLF = superior longitudinal fasciculus.

Cells show the change in FDC expressed as a percentage compared to the healthy control (HC) mean (95\% confidence interval [CI]) for each given tract either (1) going from low to high seizure frequency category (for seizure frequency) or (2) per 10 years of disease duration. For example, for the forceps major, the effect of high vs low seizure frequency was to reduce FDC by $13.85 \%$ relative to the $\mathrm{HC}$ forceps major, while the effect of 10 years of disease duration was to reduce FDC by $2.8 \%$ relative to HCs. Intracranial volume included as covariate in the models. Raw $p$ values are shown. No significant relationships were observed at a false discovery rate-corrected threshold.

was aligned across patients) into 9 named white matter tracts. Exploratory post hoc analyses were performed within the BOSD patient group to investigate the relationship between mean FDC in the 9 tracts with patient-reported seizure frequency and disease duration (in $\mathrm{R}$ notation):

tract FDC $\sim$ seizure frequency + disease duration + intracranial volume $(1)$

Seizure frequency was computed per month and categorized into low ( $<10$ seizures per month), medium (10-80 seizures per month), and high (>80 seizures per month) categories; disease duration was computed as years from age at onset to age at MRI scan.

Additional post hoc linear models were performed to explore the relationships between mean tract FD and FC separately with seizure activity. Given that FD likely reflects acute injury, we examined the association between mean tract FD and seizure frequency. Linear models were used to determine whether mean tract FD differed among low, medium, and high seizure frequency categories:

tract $\mathrm{FD} \sim$ seizure frequency + intracranial volume (2)

Because FC likely reflects chronic white matter injury, we examined the relationship between mean tract FC and the lifetime duration of epilepsy, using the following model:

tract $\mathrm{FC} \sim$ disease duration + intracranial volume (3)

Intracranial volume was included as a covariate in all models, given the likely association between FC and head size, and the inherent interdependency of FD and FC. ${ }^{8}$ BenjaminiHochberg false discovery rate (FDR) correction was performed to determine statistical significance adjusting for the multiple tract comparison for all post hoc analyses. All post hoc statistical analyses were performed in $\mathrm{R}$ (version 3.6.3).

\section{Data Availability}

The data that support the findings of this study are available upon reasonable request from the corresponding author. Data are not publicly available as they include patient data that could compromise the privacy of participants.

\section{Results}

\section{Clinical and Demographic Characteristics}

A summary of clinical and demographic characteristics of patients with BOSD is provided in Table 1. Patient and control groups were matched for age, sex, and scanner on which their MRI was acquired, and did not differ in intracranial volume.

All patients in our cohort presented with focal seizures and mean age at seizure onset was 10 years. Focal-to-bilateral tonic-clonic seizures were reported in 13/20 (65\%) patients at some point in their clinical history. Clinical data are available for each patient included in this study in Table 1 .

\section{Whole-Brain Fixel-Based Analysis}

Figure 1 shows streamline segments corresponding to fixels that exhibited a significant decrease (FWE-corrected $p$ value $<0.05$ ) in patients with BOSD when compared to healthy control participants for FD, FC, and FDC. Streamlines are colored by percentage effect decrease in the BOSD group 
Table 3 Associations Between Tract Connectivity Metrics and Clinical Disease Characteristics

\begin{tabular}{|c|c|c|c|}
\hline \multirow[b]{2}{*}{ Tract } & \multirow[b]{2}{*}{$F$ stat; model $p$ value } & \multicolumn{2}{|c|}{ Seizure frequency $^{a}$} \\
\hline & & T value & $p$ Value \\
\hline \multicolumn{4}{|c|}{ Tract FD and association with seizure frequency } \\
\hline Forceps major & $F_{3,16}=2.39 ; p=0.1069$ & -2.676 & $0.0166^{c}$ \\
\hline CC body & $F_{3,16}=1.72 ; p=0.203$ & -1.909 & 0.0744 \\
\hline Ipsilateral CST & $F_{3,16}=2.588 ; p=0.089$ & -2.619 & $0.0186^{c}$ \\
\hline Contralateral CST & $F_{3,16}=4.056 ; p=0.0254$ & -2.818 & $0.0124^{c}$ \\
\hline Ipsilateral CtT & $F_{3,16}=3.597 ; p=0.0369$ & -2.790 & $0.0131^{c}$ \\
\hline Contralateral CtT & $F_{3,16}=3.336 ; p=0.0460$ & -2.391 & 0.0294 \\
\hline Ipsilateral SLF & $F_{3,16}=0.148 ; p=0.929$ & -0.401 & 0.693 \\
\hline Contralateral SLF & $F_{3,16}=0.418 ; p=0.742$ & -1.038 & 0.315 \\
\hline \multirow[t]{2}{*}{ Ipsilateral cingulum } & $F_{3,16}=1.255 ; p=0.323$ & -1.674 & 0.114 \\
\hline & & \multicolumn{2}{|c|}{ Disease duration $^{\mathbf{b}}$} \\
\hline Tract & $F$ stat; model $p$ value & T value & $p$ Value \\
\hline \multicolumn{4}{|c|}{ Tract FC and association with disease duration } \\
\hline Forceps major & $F_{2,17}=3.491 ; p=0.0537$ & -0.979 & 0.341 \\
\hline CC body & $F_{2,17}=3.088 ; p=0.0718$ & 0.308 & 0.762 \\
\hline Ipsilateral CST & $F_{2,17}=7.616 ; p=0.0043$ & -1.637 & 0.120 \\
\hline Contralateral CST & $F_{2,17}=6.904 ; p=0.0064$ & -1.570 & 0.135 \\
\hline Ipsilateral CtT & $F_{2,17}=4.491 ; p=0.0272$ & -0.443 & 0.663 \\
\hline Contralateral CtT & $F_{2,17}=5.434 ; p=0.0149$ & -0.388 & 0.703 \\
\hline Ipsilateral SLF & $F_{2,17}=3.451 ; p=0.0055$ & 0.702 & 0.492 \\
\hline Contralateral SLF & $F_{2,17}=2.108 ; p=0.1522$ & -0.035 & 0.973 \\
\hline Ipsilateral cingulum & $F_{2,17}=3.297 ; p=0.0617$ & -1.225 & 0.237 \\
\hline
\end{tabular}

Abbreviations: CC = corpus callosum; CST = corticospinal tract; CtT = cerebellothalamic tract; FD = fiber density; SLF = superior longitudinal fasciculus.

a Seizure frequency was computed per month and categorized into low, medium, and high categories. $p$ Values are shown for the high category.

${ }^{b}$ Disease duration was calculated in y by subtracting age at seizure onset from age at the time of MRI scan. Intracranial volume was used as a covariate in

linear models for both FD and fiber bundle cross-section analyses.

'Statistical significance at the false discovery rate-corrected threshold.

when compared to controls. No fixels showed significant increases in the BOSD group when compared to controls across any of the 3 metrics.

For the FD metric, patients with BOSD exhibited substantial reductions at the forceps major and corpus callosum (at the level of sensorimotor cortices), in parts of the cerebellothalamic and corticothalamic tracts (ipsilateral > contralateral), and in the cingulum bundle ipsilateral to the BOSD lesion. Marked bilateral reductions in the FC metric were observed in motor pathways, including the corticospinal, corticothalamic, and cerebellothalamic tracts of the superior cerebellar peduncles. Commissural fiber pathways at the level of the motor cortices were also implicated, along with the ipsilateral superior longitudinal fasciculus. Reductions in the combined FDC metric were observed within both longitudinal fiber structures affected primarily by FD and motor fibers affected primarily by FC.

eFigure 1 (links.lww.com/WNL/B636) shows the same streamline segments as Figure 1, corresponding to fixels with a significant decrease in the FD, FC, and FDC metrics, this time colored by effect size (Cohen $d$ ). Medium to large effects were observed across all affected fiber pathways.

Similar findings were observed in all fixel-based metrics when lesion hemispheres were not aligned across patients (eFigure 2, links.lww.com/WNL/B636).

As described in the Supplementary Material, when comparing patients with a frontal BOSD to controls, we found significant reductions in all fixel-based metrics, which were greater in 


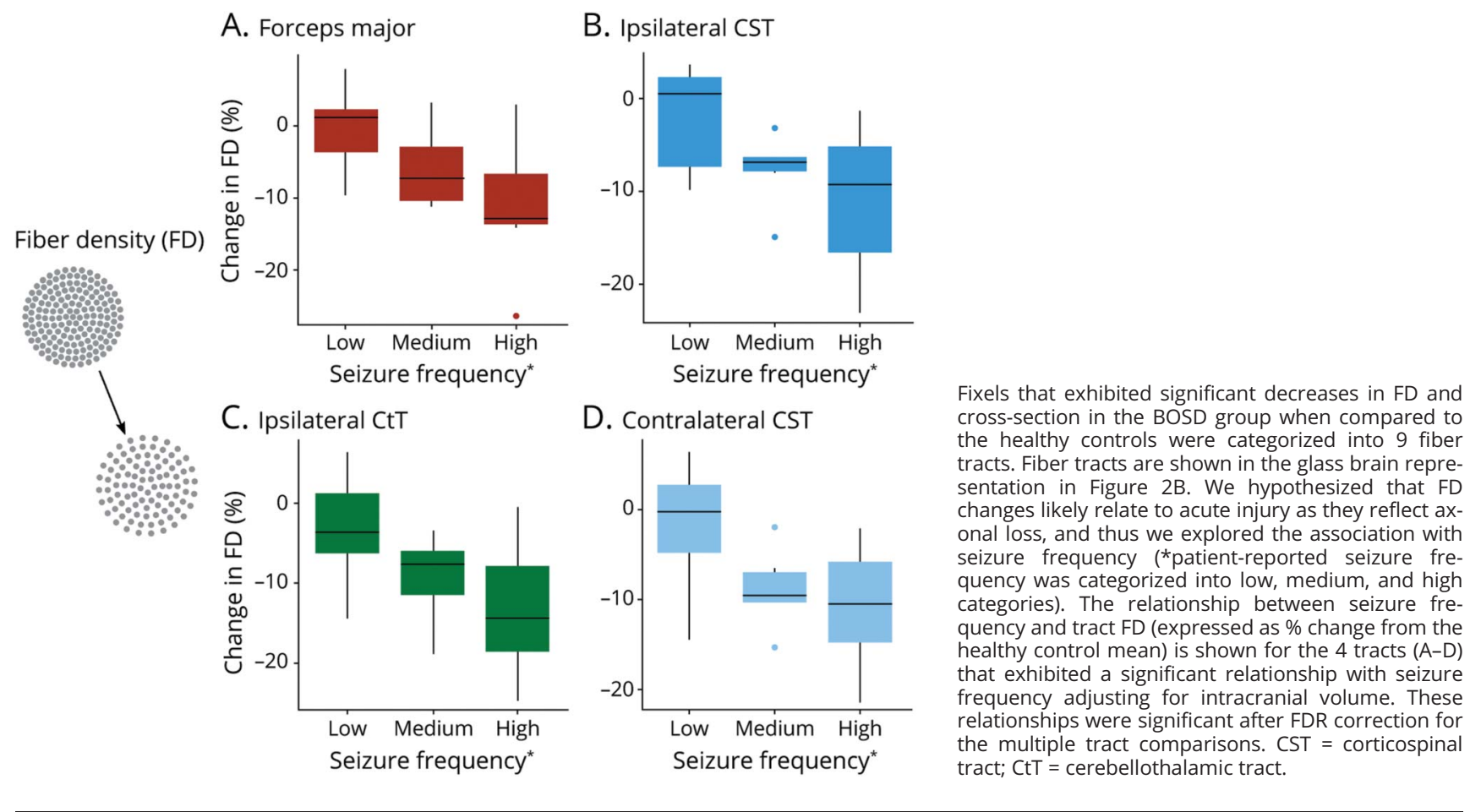

extent than was observed in the whole BOSD cohort (eFigure 3, links.lww.com/WNL/B636).

\section{Tract of Interest Analyses}

Fixels exhibiting a significant decrease in FDC in the BOSD group compared to controls were categorized into 9 tracts of interest for the purposes of exploratory post hoc analyses. These fiber pathways are shown in Figure 2, colored by direction to demonstrate the $3 \mathrm{D}$ extent of these structural connections (A), as well as by the tract to which they were assigned (B).

Exploratory post hoc analyses revealed that, of the 9 fiber tracts exhibiting significant disruptions in patients with BOSD, the largest effect of seizure frequency on FDC was evident in the forceps major (14\% decrease in FDC compared to the healthy control mean going from high to low seizure frequency), followed by a medium effect in the cerebellothalamic tracts of the superior cerebellar peduncles. The largest effect of disease duration on FDC was evident in the bilateral corticospinal tracts (ipsilateral > contralateral). No significant associations between FDC and seizure frequency or disease duration were evident at a FDR-corrected threshold. Figure 2 shows percentage change in FDC relative to the control mean in each tract going from either low to high seizure frequency category (C), or per 10 years of epilepsy duration (D), the values for which are shown in Table 2.

Table 3 shows the relationships between tract FD and seizure frequency and tract FC and disease duration. A significant effect of high seizure frequency on tract FD was observed in the forceps major, bilateral corticospinal tracts, and cerebellothalamic tracts of the superior cerebellar peduncles (Figure 3) adjusting for intracranial volume, which remained significant at an FDR-corrected level in all but the contralateral cerebellothalamic tract. As shown in Figure 3, there was a dose-dependent effect of seizure frequency of tract FD in all of these affected fiber tracts, with the FD being highest in the low seizure frequency group, followed by the medium frequency, and then the high frequency groups. No significant relationships were observed between tract FC and disease duration (Table 3).

\section{Discussion}

We assessed whether patients with a highly focal epileptogenic lesion-bottom-of-sulcus dysplasia-shared common abnormalities within white matter structural networks. By applying a whole-brain fixel-based analysis approach, we were able to identify specific white matter fiber structures that exhibited group-level differences in fiber density and crosssection in patients with BOSD when compared to control participants. Notable bilateral anomalies were observed in the sensorimotor network in our BOSD cohort, including the bilateral corticospinal and cerebellothalamic tracts, and corpus callosum at the level of the motor cortices. Reductions in fiber density and cross-section were also observed within association pathways including the bilateral superior longitudinal fasciculi and cingulum bundle ipsilateral to the BOSD 
A

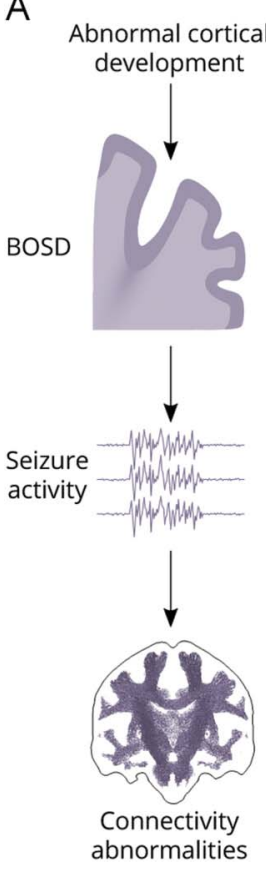

B

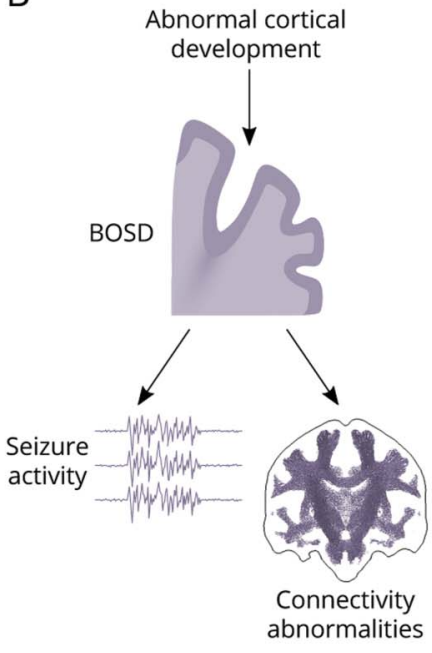

C

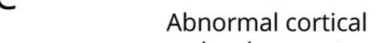
development

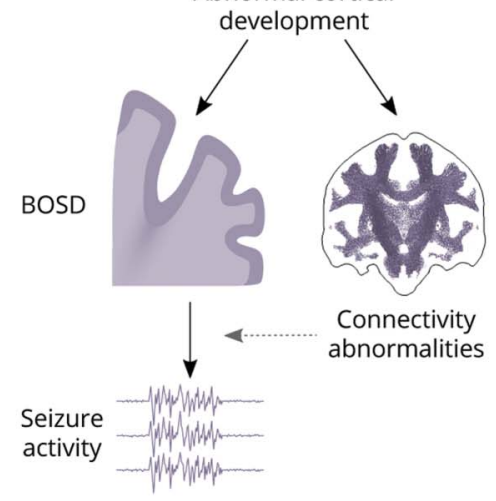

Development of BOSD results from abnormal migration and apoptosis during cortical development. Here, we refer to abnormal cortical development as the mechanisms driving the formation of a BOSD, which may relate to genetic causes. There are a number of different mechanisms by which connectivity abnormalities may arise in BOSD. (A) BOSDs are epileptogenic lesions that drive seizure activity. Connectivity abnormalities could in turn be a secondary consequence of seizure activity. (B) Connectivity abnormalities could be secondary to the development of a BOSD, but independent of seizure activity. (C) Connectivity abnormalities could arise in parallel to the development of a BOSD, potentially related to the abnormal cortical development. The epileptogenicity of BOSD lesions may be affected by being associated with abnormal structural networks (dotted arrow), although there is no direct evidence of such an effect. It is possible that a combination of all 3 of these scenarios occurs in patients with BOSD, and that abnormalities in some fiber pathways arise in line with one model, while abnormalities in others arise in line with another.

lesion, as well as in the commissural forceps major. Our findings suggest that even these highly focal lesions are characterized by widespread structural network changes. A common, bilateral structural network is likely vulnerable to damage in epilepsy associated with BOSD, and our exploratory post hoc analyses provide evidence to suggest that abnormalities within at least some fiber structures are likely related to seizure activity itself.

While there is mounting evidence for focal lesions affecting large-scale functional brain networks, ${ }^{2,3}$ there is a relative paucity of studies investigating structural connectivity abnormalities in dysplasia-related epilepsies. Previous studies investigating connectivity changes in FCDs have largely centered upon local white matter disruptions underlying the dysplastic cortex using DTI-derived metrics, ${ }^{16,17}$ in some cases reporting disruptions beyond or distal to the FCD lesion. ${ }^{18,19}$ However, in the few studies that have investigated white matter abnormalities in FCD regardless of lesion location, there is indeed evidence of common abnormalities that are shared across patients. In line with the findings of this study, bilateral reductions of DTI-based metrics in thalamocortical pathways ${ }^{20}$ and cingulum bundles ${ }^{21}$ have been reported in region of interest studies in FCD. Notably, in the only study investigating global changes in white matter in FCD, reductions in DTI-derived fractional anisotropy were reported in frontal lobe FCD in similar fiber structures to those reported here, including the forceps major, bilateral corticospinal tracts, superior longitudinal fasciculi, and cingulum bundles, among other fiber tracts. ${ }^{22}$

Of the fiber pathways found to be affected in our BOSD cohort, prominent abnormalities were evident in the sensorimotor fiber tracts. While the corticospinal tracts have been identified as affected regions in focal epilepsies, ${ }^{23}$ they are among the least affected of key fiber tracts across different epilepsy syndromes. ${ }^{24}$ We observed reductions with large effects in these fiber structures in this study (Figure 1, eFigure 1, links.lww.com/WNL/B636). Medium to large effects were also observed within fiber structures more commonly implicated in epilepsy cohorts, including the superior longitudinal fasciculi, cingulum bundle, and forceps major.

White matter tract abnormalities were not limited to the hemisphere containing the lesion, which was aligned across all patients, but were bilaterally and largely symmetrically distributed. In other focal epilepsies, such as temporal lobe epilepsy, white matter alterations are commonly reported to be more pronounced on the side ipsilateral to the seizure focus, demonstrating a lateralized effect. ${ }^{24-26}$ Moreover, the side of seizure onset has in some studies been suggested to affect the extent of observed white matter abnormalities, with left-sided lesions exhibiting greater structural abnormalities than right. ${ }^{27,28} \mathrm{We}$ did not observe prominent lateralization of white matter abnormalities in BOSD at the group level in this study, and fiber structures were similarly affected both 
ipsilateral and contralateral to the focal epileptogenic lesion. Indeed, our findings did not substantially differ when we performed group comparisons without aligning lesion hemisphere across patients (eFigure 2, links.lww.com/WNL/ B636). This symmetrical distribution of fiber tract abnormalities in BOSD suggests that these connectivity changes, rather than being a secondary consequence of the epileptogenic lesion, are driven by other factors, whether they be seizure-related or developmental.

A possible explanation for the fiber structure abnormalities is that they are a consequence of seizure activity within these structural networks (see Figure 4 for possible mechanisms). Despite controversy regarding the propensity of focal seizures to cause accelerated white matter injury, there is some evidence to suggest that focal seizures drive fiber tract reductions. ${ }^{23,25,28}$ For instance, marked fiber cross-section and density reductions were observed in our study in the forceps major, a fiber structure that has been shown by some to be transiently vulnerable to antiepileptic drug toxicity ${ }^{29}$ or drug withdrawal, ${ }^{30}$ and by others to seizure activity itself. ${ }^{31}$ Our exploratory tract of interest analyses revealed that fiber density reductions in the forceps major exhibited a significant association with seizure frequency. This suggests that the forceps major may be vulnerable to acute injury due to seizure activity.

Similarly, the corticospinal tracts have been implicated in generalized epilepsies, ${ }^{32,33}$ and DTI studies have indicated that disrupted thalamocortical circuitry is a common feature in these generalized epilepsies, ${ }^{34,35}$ consistent with functional neuroimaging evidence ${ }^{36}$ and cellular-level understanding. ${ }^{37}$ In our BOSD cohort, despite the highly focal nature of their epileptogenic lesions, patients experienced a substantial seizure burden, with many experiencing tonic-clonic seizures. In our exploratory post hoc analyses, we found FD to be significantly associated with patient-reported seizure frequency in both the ipsilateral and contralateral corticospinal and cerebellothalamic tracts (Table 3 ). In addition, the effect size of disease duration on tract FDC was greatest at the corticospinal tracts bilaterally (Figure 2). These tract of interest analyses provide preliminary evidence to suggest that seizure activity may indeed drive abnormalities within these structural networks; however, they should be considered explicitly exploratory and hypothesis-generating for future studies.

It is difficult to disentangle whether there is a causative effect of high seizure activity on fiber tract changes using a crosssectional study design. Notably, in a recent longitudinal study investigating progressive cortical thinning (presumably neuronal and axonal loss), seizure activity was not associated with the progressive thinning observed in focal epilepsy. ${ }^{38}$ Progressive atrophy has also been longitudinally observed in patients who are seizure free. ${ }^{39}$ Analogously, it is possible that seizures do not drive progressive white matter axonal loss. A higher frequency of seizures reflects a more severe epilepsy phenotype, and the observed association between fiber tract changes and seizure frequency could reflect a relationship with more severe forms of epilepsy. Alternatively, it is possible that abnormalities within fiber tracts arise during development, and individuals with more marked tract changes also experience worse seizures.

Another possible explanation for the structural anomalies in BOSD, aside from this association with seizure activity, is that they are developmental in origin and related to abnormal cortical development (Figure 4). This could be the case in some fiber structures, particularly those in which we did not observe any relationships with seizure activity. It has previously been suggested that the presence of BOSDs may in part result from disturbed neuronal connectivity, ${ }^{40}$ arising within a small window during development, when both migration and apoptosis are active. ${ }^{7}$ Long-distance connections are established early in development, and prenatal lesions that disrupt these long-range connections can not only dramatically alter cortical folding, ${ }^{41}$ but are also associated with FCD. ${ }^{42}$ Disrupted connectivity within long-distance connections has been speculated to drive sulcal abnormalities, ${ }^{40}$ and abnormal connectivity networks could arise in parallel to BOSDs.

It is also possible that a common factor during development could drive both BOSD formation as well as these long-range connectivity abnormalities. Recent genetic characterization suggests that BOSD is primarily associated with pathogenic mutations within the mTOR pathway. ${ }^{43}$ Among the numerous roles of mTOR signaling is its contribution to normal neuronal growth and neurite elongation during development, as well as regeneration following damage. ${ }^{44}$ The corticospinal tracts, which are particularly refractory to regeneration upon injury, exhibit regrowth upon enhancement of the mTOR pathway. ${ }^{45}$ The susceptibility of pathways to injury in BOSD could relate either directly to their sensitivity to mTOR signaling or due to impaired regenerative capacity due to abnormalities within the mTOR pathway following seizurerelated injury.

Structural connectivity studies in epilepsy thus far have predominantly employed DTI to investigate abnormalities within white matter structures. Whereas DTI was a pioneering method that enabled investigation of white matter structures in vivo, and has notably been applied both in the detection of focal epileptogenic lesions ${ }^{46}$ as well as in investigating their white matter connectivity, ${ }^{26}$ there are limitations in its ability to probe structural connectivity. One of the major limitations of the tensor model is its inherently voxel-averaged nature, which can pose difficulties when assessing disruptions to specific fiber pathways, and can result in misleading findings in the context of crossing-fiber populations. ${ }^{47}$ While the limitations of DTI studies have been discussed in the context of epilepsy, ${ }^{24}$ there are few studies that have moved beyond this approach to adopt advanced diffusion techniques. The FBA approach $^{8}$ applied in the present study overcomes the limitations inherent to a voxel-averaged approach such as DTI by 
moving beyond the tensor model entirely, and by performing separate comparisons on each specific fiber structure within a voxel, which enables more directly interpretable measures of structural connectivity. Recent studies using FBA have enabled identification of specific fiber structures that exhibit connectivity changes in other focal epilepsies. ${ }^{25,48}$

Although there are advantages in the methodology used in this work, there are a number of limitations to our study. We explored the potential association of tract-specific abnormalities with seizure frequency and disease duration, finding a possible effect from recurrent seizures; however, these post hoc analyses must be considered hypothesis-generating, and we are limited by a relatively small cohort size. Given the long period of time over which patients were recruited for this study, there was variability in the scanner and DWI sequence across patients. Crucial parameters such as b-value were consistent across all participants, and scanner type was included as a nuisance regressor in our primary analyses; however, it would be preferable if scanner and sequence harmonization could be achieved for future studies to minimize potential sources of variance. Our patient cohort included only adolescents and adults, many of whom had a long history of epilepsy and antiepileptic drug use. We did not assess associations with antiepileptic drug use in this study and there is some evidence suggesting that some drugs may affect white matter in focal epilepsy, ${ }^{49}$ although medicationrelated MRI abnormalities are sometimes reported to be transient. ${ }^{50}$ In this context, longitudinal studies are needed to assess the effects of chronic epilepsy and antiepileptic drug use on these fiber tracts, the mechanisms for which can be otherwise difficult to unravel in a cross-sectional study design. Future studies in pediatric cohorts are needed to differentiate developmental from seizure-related disruptions of fiber pathways and clarify whether abnormal structural networks and the cognitive consequences with which they are associated are present at the onset of seizures or are the result of seizure activity itself.

The findings of this study demonstrate extensive, bilateral abnormalities within structural networks in patients with epilepsy associated with a BOSD. For some fiber tracts, the extent of abnormality is related to seizure activity; however, we have not excluded the contribution of other factors such as abnormal development to these tract abnormalities. If the white matter abnormalities observed in this cohort relate to chronic epilepsy, this provides strong support for early seizure control to inhibit the progression of fiber tract disruptions in BOSD through surgery, particularly given the excellent surgical outcomes in this disorder.

\section{Acknowledgment}

The authors thank the participants for their involvement in this study. The Florey Institute of Neuroscience and Mental Health acknowledges support from the Victorian government and in particular funding from the Operational Infrastructure Support Grant. The authors acknowledge the facilities and scientific and technical assistance of the National Imaging Facility, a National Collaborative Research Infrastructure Strategy (NCRIS) capability, at the Florey Institute of Neuroscience and Mental Health.

\section{Study Funding}

Supported by the National Health and Medical Research Council (NHMRC) of Australia and an NHMRC grant (1091593).

\section{Disclosure}

The authors report no disclosures relevant to the manuscript. Go to Neurology.org/ $\mathrm{N}$ for full disclosures.

\section{Publication History}

Received by Neurology April 28, 2021. Accepted in final form October 15, 2021.

\begin{tabular}{lll} 
Appendix & Authors & \\
\hline Name & Location & Contribution \\
\hline $\begin{array}{l}\text { Remika Mito, } \\
\text { PhD }\end{array}$ & $\begin{array}{l}\text { Florey Institute of } \\
\text { Neuroscience and Mental } \\
\text { Health, Melbourne }\end{array}$ & $\begin{array}{l}\text { Designed and conceptualized } \\
\text { study; analyzed the data; } \\
\text { drafted manuscript for } \\
\text { intellectual content }\end{array}$ \\
\hline $\begin{array}{l}\text { David N. } \\
\text { Vaughan, } \\
\text { MBBS, PhD }\end{array}$ & $\begin{array}{l}\text { Florey Institute of } \\
\text { Neuroscience and Mental }\end{array}$ & $\begin{array}{l}\text { Interpreted the data; revised } \\
\text { the manuscript for intellectual } \\
\text { content }\end{array}$ \\
\hline $\begin{array}{l}\text { Mira } \\
\text { Semmelroch, } \\
\text { MSc }\end{array}$ & $\begin{array}{l}\text { Florey Institute of } \\
\text { Neuroscience and Mental } \\
\text { Health, Melbourne }\end{array}$ & $\begin{array}{l}\text { Major role in acquisition of } \\
\text { data }\end{array}$ \\
\hline $\begin{array}{l}\text { Alan } \\
\text { Connelly, } \\
\text { PhD }\end{array}$ & $\begin{array}{l}\text { Florey Institute of } \\
\text { Neuroscience and Mental } \\
\text { Health, Melbourne }\end{array}$ & $\begin{array}{l}\text { Interpreted the data; revised } \\
\text { the manuscript for intellectual } \\
\text { content }\end{array}$ \\
\hline $\begin{array}{l}\text { Graeme D. } \\
\text { Jackson, MD, } \\
\text { FRACP }\end{array}$ & $\begin{array}{l}\text { Florey Institute of } \\
\text { Neuroscience and Mental } \\
\text { Health, Melbourne }\end{array}$ & $\begin{array}{l}\text { Interpreted the data; revised } \\
\text { the manuscript for intellectual } \\
\text { content }\end{array}$ \\
\hline
\end{tabular}

\section{References}

1. Bernasconi A. Connectome-based models of the epileptogenic network: a step towards epileptomics? Brain. 2017;140(10):2525-2527.

2. Tavakol S, Royer J, Lowe AJ, et al. Neuroimaging and connectomics of drug-resistant epilepsy at multiple scales: from focal lesions to macroscale networks. Epilepsia. 2019; 60(4):593-604.

3. Hong S-J, Lee H-M, Gill R, et al. A connectome-based mechanistic model of focal cortical dysplasia. Brain. 2019;142(3):688-699.

4. Hong S-J, Bernhardt BC, Gill RS, Bernasconi N, Bernasconi A. The spectrum of structural and functional network alterations in malformations of cortical development. Brain. 2017;140(8):2133-2143.

5. Barkovich AJ, Kuzniecky RI, Jackson GD, Guerrini R, Dobyns WB. A developmental and genetic classification for malformations of cortical development. Neurology. 2005;65(12): 1873-1887.

6. Harvey AS, Mandelstam SA, Maixner WJ, et al. The surgically remediable syndrome of epilepsy associated with bottom-of-sulcus dysplasia. Neurology. 2015;84(20):2021-2028.

7. Hofman PAM, Fitt GJ, Harvey AS, Kuzniecky RI, Jackson G. Bottom-of-sulcus dysplasia: imaging features. Am J Roentgenol. 2011;196(4):881-885.

8. Raffelt DA, Tournier J-D, Smith RE, et al. Investigating white matter fiber density and morphology using fixel-based analysis. Neuroimage. 2017;144:58-73.

9. Tournier J-D, Smith RE, Raffelt DA, et al. MRtrix3: a fast, flexible and open software framework for medical image processing and visualisation. Neuroimage. 2019;202:116137.

10. Dhollander T, Connelly A. A novel iterative approach to reap the benefits of multitissue CSD from just single-shell $(+b=0)$ diffusion MRI data. In: 24th International Society of Magnetic Resonance in Medicine; 2016.

11. Raffelt D, Tournier J-D, Fripp J, Crozier S, Connelly A, Salvado O. Symmetric diffeomorphic registration of fiber orientation distributions. Neuroimage. 2011;56(3): 1171-1180. 
12. Smith RE, Tournier J-D, Calamante F, Connelly A. SIFT: spherical-deconvolution informed filtering of tractograms. Neuroimage. 2013;67:298-312.

13. Raffelt D, Tournier J-D, Rose S, et al. Apparent fiber density: a novel measure for the analysis of diffusion-weighted magnetic resonance images. Neuroimage. 2012;59(4): 3976-3994.

14. Mori S, Wakana S, Van Zijl PCM, Nagae-Poetscher LM. MRI Atlas of Human White Matter. Elsevier; 2005.

15. Raffelt DA, Smith RE, Ridgway GR, et al. Connectivity-based fixel enhancement: whole-brain statistical analysis of diffusion MRI measures in the presence of crossing fibers. Neuroimage. 2015;117:40-55.

16. Lee S-K, Kim DI, Mori S, et al. Diffusion tensor MRI visualizes decreased subcortical fiber connectivity in focal cortical dysplasia. Neuroimage. 2004;22(4):1826-1829.

17. Gross DW, Bastos A, Beaulieu C. Diffusion tensor imaging abnormalities in focal cortical dysplasia. Can J Neurol Sci. 2005;32(4):477-482.

18. Eriksson Sh, Rugg-Gunn FJ, Symms MR, Barker GJ, Duncan JS. Diffusion tensor imaging in patients with epilepsy and malformations of cortical development. Brain. 2001;124(3):617-626.

19. Widjaja E, Zarei Mahmoodabadi S, Otsubo H, et al. Subcortical alterations in tissue microstructure adjacent to focal cortical dysplasia: detection at diffusion-tensor MR imaging by using magnetoencephalographic dipole cluster localization. Radiology. 2009;251(1):206-215.

20. Rezayev A, Feldman HA, Levman J, Takahashi E. Bilateral thalamocortical abnormalities in focal cortical dysplasia. Brain Res. 2018;1694:38-45.

21. Campos BM, Coan AC, Beltramini GC, et al. White matter abnormalities associate with type and localization of focal epileptogenic lesions. Epilepsia. 2015;56(1): 125-132.

22. Fonseca VC, Yasuda CL, Tedeschi GG, Betting LE, Cendes F. White matter abnormalities in patients with focal cortical dysplasia revealed by diffusion tensor imaging analysis in a voxelwise approach. Front Neurol. 2012;3:121.

23. Slinger G, Sinke MRT, Braun KPJ, Otte WM. White matter abnormalities at a regional and voxel level in focal and generalized epilepsy: a systematic review and metaanalysis. Neuroimage Clin. 2016;12:902-909.

24. Hatton SN, Huynh KH, Bonilha L, et al. White matter abnormalities across different epilepsy syndromes in adults: an ENIGMA-Epilepsy study. Brain. 2020;143(8) 2454-2473.

25. Vaughan DN, Raffelt D, Curwood E, et al. Tract-specific atrophy in focal epilepsy: disease, genetics, or seizures? Ann Neurol. 2017;81(2):240-250.

26. Otte WM, van Eijsden P, Sander JW, Duncan JS, Dijkhuizen RM, Braun KPJ. A metaanalysis of white matter changes in temporal lobe epilepsy as studied with diffusion tensor imaging. Epilepsia. 2012;53(4):659-667.

27. Ahmadi ME, Hagler DJ, McDonald CR, et al. Side matters: diffusion tensor imaging tractography in left and right temporal lobe epilepsy. Am J Neuroradiol. 2009;30(9): 1740-1747.

28. Keller SS, Schoene-Bake J-C, Gerdes JS, Weber B, Deppe M. Concomitant fractional anisotropy and volumetric abnormalities in temporal lobe epilepsy: cross-sectional evidence for progressive neurologic injury. PLoS One. 2012;7(10):e46791.

29. Kim SS, Chang K-H, Kim ST, et al. Focal lesion in the splenium of the corpus callosum in epileptic patients: antiepileptic drug toxicity? Am J Neuroradiol. 1999;20(1): 125-129.
30. Gürtler S, Ebner A, Tuxhorn I, Ollech I, Pohlmann-Eden B, Woermann FG. Transient lesion in the splenium of the corpus callosum and antiepileptic drug withdrawal. Neurology. 2005;65(7):1032-1036.

31. Oster J, Doherty C, Grant PE, Simon M, Cole AJ. Diffusion-weighted imaging abnormalities in the splenium after seizures. Epilepsia. 2003;44(6):852-854.

32. Liu M, Concha L, Beaulieu C, Gross DW. Distinct white matter abnormalities in different idiopathic generalized epilepsy syndromes. Epilepsia. 2011;52(12): 2267-2275.

33. Jackson G, Sadeghian F, Carney P, Raffelt D, Calamante F, Connelly A. Structural connectivity changes in refractory childhood absence epilepsy. In: International Society for Magnetic Resonance in Medicine (ISMRM); 2016.

34. O'Muircheartaigh J, Vollmar C, Barker GJ, et al. Abnormal thalamocortical structural and functional connectivity in juvenile myoclonic epilepsy. Brain. 2012;135(12): 3635-3644.

35. Groppa S, Moeller F, Siebner H, et al. White matter microstructural changes of thalamocortical networks in photosensitivity and idiopathic generalized epilepsy. Epilepsia. 2012;53(4):668-676.

36. Gotman J, Grova C, Bagshaw A, Kobayashi E, Aghakhani Y, Dubeau F. Generalized epileptic discharges show thalamocortical activation and suspension of the default state of the brain. Proc Natl Acad Sci. 2005;102(42):15236-15240.

37. Blumenfeld H. Cellular and network mechanisms of spike-wave seizures. Epilepsia. 2005;46:21-33.

38. Galovic M, van Dooren VQH, Postma TS, et al. Progressive cortical thinning in patients with focal epilepsy. JAMA Neurol. 2019;76(10):1230-1239.

39. Alvim MKM, Coan AC, Campos BM, et al. Progression of gray matter atrophy in seizure-free patients with temporal lobe epilepsy. Epilepsia. 2016;57(4):621-629.

40. Besson P, Andermann F, Dubeau F, Bernasconi A. Small focal cortical dysplasia lesions are located at the bottom of a deep sulcus. Brain. 2008;131(12):3246-3255.

41. Van Essen DC. A tension-based theory of morphogenesis and compact wiring in the central nervous system. Nature. 1997;385(6614):313-318

42. Krsek P, Jahodova A, Maton B, et al. Low-grade focal cortical dysplasia is associated with prenatal and perinatal brain injury. Epilepsia. 2010;51(12):2440-2448.

43. Lee WS, Stephenson SEM, Pope K, et al. Genetic characterisation identifies bottomof-sulcus dysplasia as an mTORopathy. Neurology. 2020;95(18):e2542-e2551.

44. Wei X, Luo L, Chen J. Roles of mTOR signaling in tissue regeneration. Cells. 2019; 8(9): 1075 .

45. Liu K, Lu Y, Lee JK, et al. PTEN deletion enhances the regenerative ability of adult corticospinal neurons. Nat Neurosci. 2010;13(9):1075-1081.

46. Rugg-Gunn FJ, Eriksson SH, Symms MR, Barker GJ, Duncan JS. Diffusion tensor imaging of cryptogenic and acquired partial epilepsies. Brain. 2001;124(3):627-636

47. Mito R, Raffelt D, Dhollander T, et al. Fiber-specific white matter reductions in Alzheimer's disease and mild cognitive impairment. Brain. 2018;141(3):888-902.

48. Bauer T, Ernst L, David B, et al. Fixel-based analysis links white matter characteristics, serostatus and clinical features in limbic encephalitis. Neuroimage Clin 2020;27: 102289.

49. Pardoe HR, Berg AT, Jackson GD. Sodium valproate use is associated with reduced parietal lobe thickness and brain volume. Neurology. 2013;80(20):1895-1900.

50. Wheless JW, Carmant L, Bebin M, et al. Magnetic resonance imaging abnormalities associated with vigabatrin in patients with epilepsy. Epilepsia. 2009;50(2):195-205. 


\section{Neurology}

\section{Bilateral Structural Network Abnormalities in Epilepsy Associated With Bottom-of-Sulcus Dysplasia}

Remika Mito, David N. Vaughan, Mira Semmelroch, et al.

Neurology 2022;98;e152-e163 Published Online before print October 21, 2021

DOI 10.1212/WNL.0000000000013006

This information is current as of October 21, 2021

\section{Updated Information \&} Services

References

Subspecialty Collections

Permissions \& Licensing

Reprints including high resolution figures, can be found at: http://n.neurology.org/content/98/2/e152.full

This article cites 47 articles, 7 of which you can access for free at: http://n.neurology.org/content/98/2/e152.full\#ref-list-1

This article, along with others on similar topics, appears in the following collection(s):

All Epilepsy/Seizures

http://n.neurology.org/cgi/collection/all_epilepsy_seizures

Cortical dysplasia

http://n.neurology.org/cgi/collection/cortical_dysplasia

DWI

http://n.neurology.org/cgi/collection/dwi

MRI

http://n.neurology.org/cgi/collection/mri

Information about reproducing this article in parts (figures,tables) or in its entirety can be found online at:

http://www.neurology.org/about/about_the_journal\#permissions

Information about ordering reprints can be found online:

http://n.neurology.org/subscribers/advertise

Neurology ${ }^{\circledR}$ is the official journal of the American Academy of Neurology. Published continuously since 1951, it is now a weekly with 48 issues per year. Copyright Copyright (C) 2021 The Author(s). Published by Wolters Kluwer Health, Inc. on behalf of the American Academy of Neurology.. All rights reserved. Print ISSN: 0028-3878. Online ISSN: 1526-632X.

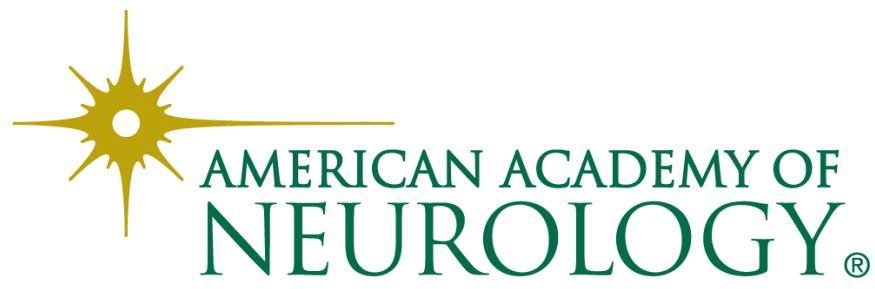

